\title{
A NOTE ON RINGS WITH A WEAK ALGORITHM
}

\author{
BY R. E. WILLIAMS
}

\section{Communicated by Saunders MacLane, March 6, 1969}

Introduction. The principal examples of rings with a weak algorithm in [3] are tensor rings on $K$-bimodules, $K$ a division ring. By introducing two maps $S: K \rightarrow K_{n}, D: K \rightarrow V_{n}(K)$ which satisfy the Ore-like conditions: $(\alpha \beta)^{S}=\alpha^{S} \beta^{S},(\alpha \beta)^{D}=\alpha^{D} \beta^{S}+\alpha \beta^{D}$, all $\alpha, \beta$ in $K$, various rings with the weak algorithm are obtained from tensor rings. Here, $n=|X|, X$ a right $K$-basis of the given $K$-bimodule $N$. A separation of this class into families is easily observed. To deal with arbitrary rings with a weak algorithm, in $\$ 2$ a class of rings extending the class of tensor rings is defined. As for tensor rings, other rings with the weak algorithm (all of them, in fact) are obtained from these rings and separated into families. It is shown then that each ring in the class extending the class of tensor rings is embeddable in a division ring and this raises a central question: Can the embedding theorem be generalized so as to apply to the associated classes?

Throughout, ring shall mean associative ring with $1, K$ shall denote a division ring, $X$ a generating set for the ring and $x_{I}$ the monomial $x_{i_{1}} \cdots x_{i_{n}}$ where $I=\left(i_{1}, \cdots, i_{n}\right)$. Although the proofs are carried out using right polynomials, right $K$-modules, etc., the results in [3] insure that there is a left-right symmetry.

1. Families associated with tensor rings. Let $R$ be a ring with the weak algorithm, let $K$ be the underlying division ring and let $X=\left\{x_{\lambda}: \lambda \in \Lambda\right\}$ be an $R$-independent generating set of $R$. We set $N=\left\{\sum x_{\lambda} \alpha_{\lambda}: x_{\lambda} \in X\right\}$ and for the moment restrict ourselves to rings for which $M=N+K$ is a $K$-bimodule. Our first result is

THEOREM 1.1 Let $R$ be a ring with a weak algorithm for which $M$ is a $K$-bimodule. The structure of $M$, and hence of $R$, is completely determined by two maps $S: K \rightarrow K_{n}, D: K \rightarrow V_{n}(K), n=|Z|, Z$ a right $K$ basis of $N$, which satisfy $(\alpha \beta)^{S}=\alpha^{S} \beta^{S},(\alpha \beta)^{D}=\alpha^{D} \beta^{S}+\alpha \beta^{D}, K \approx K^{S}$. Moreover, any such $S, D$ yield a ring with the weak algorithm.

As each element of $R$ has a unique expression

$$
\sum x_{I} \alpha_{I} \quad \text { (a.a. } \alpha_{I}=0, \quad I \text { over all ordered finite subsets of } \Lambda \text { ) }
$$

as a right polynomial on $X$, the structure of $M$ determines that of $R$. Next order $X:\left\{x_{1}, x_{2}, \cdots\right\}$ and for each $x_{i}$ set 


$$
\beta x_{i}=\sum_{j} x_{j} \beta_{j i}+\beta^{d_{i}}, \quad\left(\beta, \beta_{j i}, \beta^{d_{i}} \in K\right) .
$$

The associativities $\alpha\left(\beta x_{i}\right)=(\alpha \beta) x_{i}$ yield that $\alpha \rightarrow \alpha^{S}=\left(\alpha_{j i}\right), \alpha \rightarrow \alpha^{D}$ $=\left(\alpha^{d_{1}}, \cdots\right)$ satisfy the above conditions. Given such $S$ and $D$ the construction of $R$ is immediate, the weak algorithm holding essentially because of the unique form (1).

The notation $(|X|, K, S, D)$ shall be used for a ring for which $M$ is a $K$-bimodule. Observe the

Lемма 1.2. $(|X|, K, S, D)$ is a tensor ring if and only if $D=0$.

A separation of this class into families is now obtained.

Theorem 1.3. If $C$ denotes the class of all rings with the weak algorithm for which $M$ is a $K$-bimodule then $C$ consists of nonoverlapping families $\left(|X|, K, S, D_{a}: a \in A\right)$, each containing exactly one tensor ring.

2. The general case. The requirement that $M$ be a $K$-bimodule need not hold in general [3], [1, p. 37]. If we replace $N$ by the $K$ bimodule generated by $X: N=\left\{\sum \alpha_{\lambda} x_{\lambda} \beta_{\lambda}: \alpha_{\lambda}, \beta_{\lambda} \in K, \lambda \in \Lambda\right\}$ and again let $M=N+K, Y$ be a right $K$-basis of $M$ it is clear that as above maps $S: K \rightarrow K_{n}, D: K \rightarrow V_{n}(K)$ are defined where $n=|Y|$. The analogue of Theorem 1.1 is

THEOREM 2.1. Let $R$ be a ring with a weak algorithm with $M$ the underlying $K$-bimodule. The structure of $M$, and hence of $R$, is completely determined by two maps $S: K \rightarrow K_{n}, D: K \rightarrow V_{n}(K)$ which satisfy $(\alpha \beta)^{S}=\alpha^{S} \beta^{S},(\alpha \beta)^{D}=\alpha^{D} \beta^{S}+\alpha \beta^{D}, K \approx K^{S}$, where $n=|Y|, Y$ a right $K$-basis of $M$. Moreover, any such $Y, S, D$ determines a ring with the weak algorithm.

As Lemma 1.2 may suggest, it is possible to substitute the condition $D=0$ in place of requiring that our ring be a tensor ring. This yields the following

TheOREM 2.2. Let $C$ denote the class of rings with the weak algorithm. $C$ consists of nonoverlapping families ( $\left.Y, K, S, D_{a}: a \in A\right)$, each containing exactly one ring for which $D_{a}=0$.

Here of course $|Y|$ need not equal $|X|+1$; it does so, in fact, precisely when $D_{a}=0$ yields a tensor ring.

3. The embedding theorem. By using Cohn's embedding theorem [2] we establish that the rings $(Y, K, S, O)$ may be embedded in a division ring. In the theorem below we assume there is an $x_{0} \in X$ for which $\alpha x_{0}=x_{0} \alpha$ for all $\alpha$ in $K$. This is no restriction for our ring $R$ is a subring of such a ring. The proof is even easier if it is assumed that there are two such elements in $X$. 
THEOREM 3.1. Let $R$ be a ring with the weak algorithm for which $D=0$. Then $R$ is embeddable in a division ring.

Take $|X|>1$, give $R$ the filtration

$$
R_{n}=\left\{\sum\left(x_{I}-x_{J}\right) \alpha:|I|=|J| \geqq n\right\}
$$

and define $\bar{v}$ by $\bar{v}(0)=\infty, \bar{v}(a)=\min \left\{n: a \notin R_{n+1}\right\}, a \neq 0$. $\bar{v}$ is a valuation except that $\bar{v}(a b)=\bar{v}(a)+\bar{v}(b)$ is insured only for $a \in R_{1}$. Thus the embedding is done with $R_{1}$ and $R$ is recovered via $x_{\lambda}=\left(x_{\lambda} x_{I}-x_{\lambda} x_{J}\right)$ $\cdot\left(x_{I}-x_{J}\right)^{-1}$. It is required to show that $f_{a, b}(x, y)=\bar{v}(a x-b y)-\bar{v}(a x)$ is unbounded above for $a, b \neq 0 \in R_{1}$. A key observation is that it is sufficient to establish this only for $x, y \in R$.

Induction on $l(a)=$ number of terms in the unique form (1) for $a$ is used. If $l(a)<2, a=0$ and if $l(a)=2$ an easy induction on $l(b)$ yields the result. Now let $l(a)=k>2$. Write $a=a_{1}+a_{0}, l\left(a_{0}\right)=2$ and select $x, y$ such that

$$
a_{1} x-b v=a x-b y-a_{0} x \in R_{t}, \quad t=\bar{v}(b y)+n .
$$

For some $g \in K\left[x_{0}\right] \subset R, a_{0} x-g \in R_{t}$ so $a x-b y-g \in R_{t}$. We easily show that for some $u, w \neq 0 \in R, g u-a w \in R_{t}$ using the same steps as for $a, b$ with $g_{1}$ replacing $g$ and then the common right multiple property of $K\left[x_{0}\right]$. Hence

$$
(a x-b y-g) u+g u-a w=a(x u-w)-b y u \in R_{t} .
$$

An appropriate choice of $w$ establishes the theorem.

\section{REFERENCES}

1. G. M. Bergman, Commuting elements in free algebras and related topics in ring theory, Ph.D. Thesis, Harvard University, Cambridge, Mass., 1967 (unpublished).

2. P. M. Cohn, On the embedding of rings in skew fields, Proc. London Math. Soc. (3) 11 (1961), 511-530.

3. - Rings with a weak algorithm, Trans. Amer. Math. Soc. 109 (1963), 332356.

Kansas State University, Manhattan, Kansas 66502 